\title{
ANOMALOUS NON-LINEAR DIELECTRIC BEHAVIOUR OF LATGS CRYSTALS*
}

\author{
R. $\mathrm{CACH}^{a}$, J. MRóz ${ }^{b}$ AND D. HADEN ${ }^{a}$ \\ a Institute of Experimental Physics, University of Wrocław, Cybulskiego 36, \\ 50-205 Wrocław, Poland \\ ${ }^{b}$ Institute of Physics, Technical University of Wrocław, \\ Wybrzezie S. Wyspiańskiego 27, 50-317 Wrocław, Poland
}

(Received February 19, 1991)

The dielectric permittivity in a biasing electric field and also the spontaneous polarization of LATGS crystals are measured near the ferroelectric phase transition. It is demonstrated that L-alanine admixtures change the parameters of dielectric state equation. It is shown that the bias field value decreases while approaching the phase transition temperature from the paraelectric phase. The effective non-linearity coefficient $B$, near $T_{\mathrm{C}}$, depends on electric field direction. This anomalous non-linear behaviour can be explained assuming asymmetric bias field dependence on external electric field.

PACS numbers: 77.80.Bh, 77.20 +y

\section{Introduction}

The partial substitution of $\mathrm{L}$-alanine for glycine induces an internal bias field $E_{\mathrm{B}}$ in TGS crystals. From the first reports on that subject [1, 2] LATGS crystals have been extensively studied because of their application as pyroelectric detectors. The value of $E_{\mathrm{B}}$ is associated with the concentration of $\mathrm{L}$-alanine incorporated in the crystal [3, 4], however its origin is not completely clear. According to the model prposed by Keve et al. [2] alanine molecules replace glycine I molecules in a host lattice resulting in the stable polarization along the ferroelectric axis. The dielectric hysteresis loop shows considerable unipolarity and displacement along the electric field axis. The latter is considered as an effect of the internal bias field which can be treated as a measure of the polarization direction stability. An eguivalence of $E_{\mathrm{B}}$ and an external electric field has been shown comparing their

*This work was partially sponsored by CPBP 01.12. 
effect on such physical quantities as maximum dielectric permittivity value $\varepsilon_{\mathrm{m}}$ [3] and temperature of its appearing $T_{m}[3,5]$, spontaneous polarization [6], or specific heat [7]. However, it seems that this equivalence exists for electric field values higher than $E_{\mathrm{B}}$ [8]. The spatial distribution of L-alanine content of LATGS crystals is inhomogeneous and it has a very large influence on the values of $E_{\mathrm{B}}$ [4, $5,9,10]$. The correlation between the growth asymmetry, the sense of spontaneous polarization vector and distribution of L-alanine was also studied [10,11]. LATGS crystal develop habit which is asymmetric about the (010) plane. The degree of asymmetry increases with increasing L-alanine content. The crystal form of highly doped LATGS has the polar symmetry 2 which coincides with the point group of pure TGS in the ferroelectric phase [11]. The empirical dependence between the L-alanine content of the crystal and the internal bias field was found by Koralewski et al. [4]. From various experiments the temperature dependence of internal bias field has been concluded [13-16]. The results, however, are not univocal but they show that the mechanism of polarization stabilization in-LATGS crystals is rather complicated. The effect of L-alanine admixture in TGS can not be characterized only by constant internal bias field, especially for low alanine content in the crystal.

The extensive literature concerning LATGS crystals lacks the quantitative analysis of crystals properties from the view point of classical thermodynamic theory. For the description of the temperature dependence of LATGS spontaneous polarization $\mathrm{K}$. Imai and $\mathrm{H}$. Ishida [6] used the dielectric state equation coefficients estimated by Triebwasser for pure TGS. However considering LATGS crystal as TGS crystal at $E_{\mathrm{B}}$ field seems to be rather risky. It is known that organic admixtures in TGS change the Curie-Weiss constant [12] and in consequence also the first coefficient $\left(A_{0}\right)$ in the dielectric state equation:

$$
T=A_{0}\left(T-T_{\mathrm{C}}\right) D+B D^{3}+C D^{5},
$$

where $T_{\mathrm{C}}$ is the Curie temperature, $D$-electric displacement, $A_{0}, B$ and $C$-coefficients.

The L-alanine admixture influence on temperature dependence of spontaneous polarization can be connected with the change of other coefficients, particularly with the change of the non-linearity coefficient $B$. A crystal quality has also an influence on the temperature dependence of $B$ coefficient [17].

In the present paper we discuss the non-linear dielectric properties and the $E_{\mathrm{B}}$ behaviour for LATGS crystals of relatively low L-alanine concentration.

\section{Samples and measurement}

Two LATGS crystals were grown at $303 \mathrm{~K}$ from solutions of various concentration of L-alanine. The samples were cut out from (001) growth pyramid. Taking into consideration the results from [4] we can assume that L-alanine contents of the studied samples was 0.04 weight \% (crystal I) and 0.05 weight \% (crystal II). The silver paste electrodes were used for dielectric measurements.

The spontaneous polarization $P_{\mathrm{S}}$ was determined from ferroelectric hysteresis loops using the Diamant, Drenck and Pepinsky circuit at $50 \mathrm{~Hz}$. Dielectric permit- 
tivity as a function of temperature, at d.c. électric field was measured using RLC meter, working with frequency of $1 \mathrm{kHz}$ and a voltage not higher than $1 \mathrm{~V}$. The measurement was made on cooling at a rate of $0.3 \mathrm{deg} / \mathrm{min}$. In the paraelectric phase the field dependence of dielectric permittivity was measured. The sample polarization was modulated applying low frequency field $(0.01$ and $0.005 \mathrm{~Hz})$ of the amplitude of about $200 \mathrm{kV} / \mathrm{m}$. The temperature was stabilizeed to within $0.02 \mathrm{~K}$.

\section{Results}

In the Fig. 1 the temperature dependence of reciprocal dielectric constant is presented. For both crystals, above $T_{\mathrm{C}}$, the Curie-Weiss law was well obeyed. For the crystal I the Curie-Weiss constant was $3493 \mathrm{~K}^{-1}$, whereas it was lower for the crystal II amounting to $2753 \mathrm{~K}^{-1}$. The latter value is close to that obtained by Gaffar et al. [12]. The coefficient $A_{0}$ values estimated from these data for crystals $I$ and II are $3.23 \times 10^{7}$ and $4.10 \times 10^{7} \mathrm{VmC}^{-1} \mathrm{~K}^{-1}$ respectively.

Measurements of the dielectric permittivity as a function of temperature for several d.c. fields were carried out. From the dependence of the dielectric permittivity maximum $\varepsilon_{\mathrm{m}}$ on electric field the internal bias field and non-linearity coefficient $B$ can be determined. If the dielectric state equation (1) can be limited to two first terms only, the following relation is fulfilled [18]:

$$
\varepsilon_{\mathrm{m}}^{-3 / 2}=3^{3 / 2} 2^{-1 / 2} B^{1 / 2} E .
$$

In the Fig. 2 the $-3 / 2$ power of $\varepsilon_{m}$ as a function of electric field for the crystal
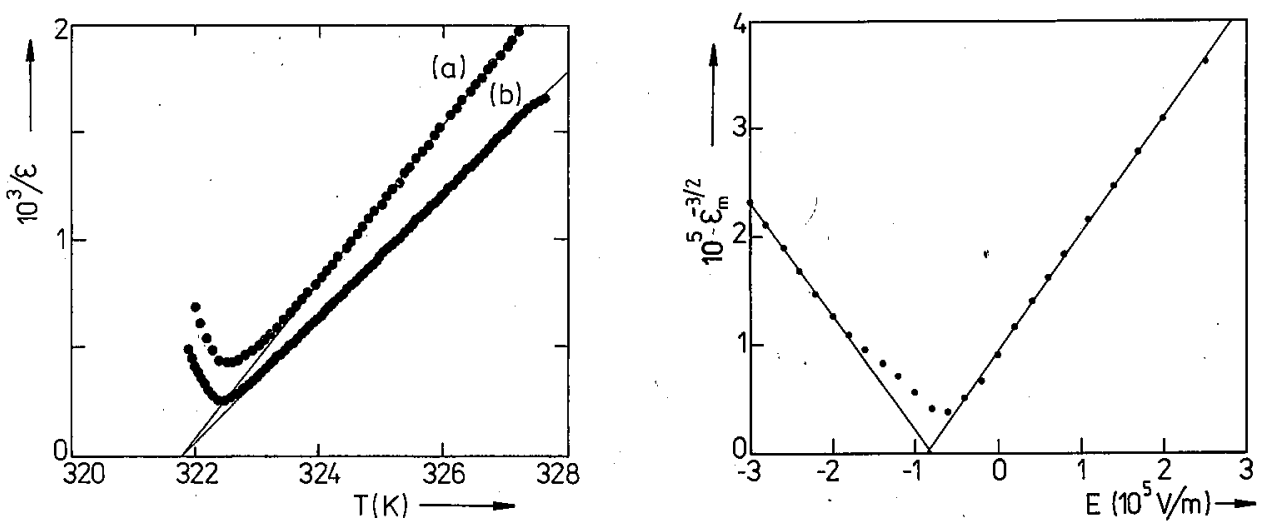

Fig. 1. The reciprocal dielectric permittivity for crystal I (b) and II (a).

Fig. 2. $-3 / 2$ power of $\varepsilon_{\mathrm{m}}$ as a function of electric field (crystal II).

II is presented. The dependence is linear except for some field range around the $-E_{\mathrm{B}}$ value. It is characteristic of LATGS crystals that even if $E_{\mathrm{B}}$ is compensated by external electric field, $\varepsilon_{\mathrm{m}}$ is lower than that for pure TGS. The linear parts of 
the dependence under discussion show on the $E$-axis the internal bias field value. We have obtained $50 \mathrm{kV} / \mathrm{m}$ and $83 \mathrm{kV} / \mathrm{m}$ for crystals I and II respectively.

From the linear dependence of $\varepsilon_{\mathrm{m}}^{-3 / 2}$ on electric field $E$, the non-linearity coefficient $B$ was estimated. We have obtained $(7.6 \pm 0.2) \times 10^{11}$ and $(12.1 \pm 0.6) \times 10^{11} \times$ $\mathrm{Vm}^{5} \mathrm{C}^{-3}$ for crystals I and II respectively. The first value (crystal I) is close to that of pure TGS, but the second value (crystal II) is considerably higher. To investigate in detail the non-linear dielectric properties, the dielectric permittivity as a function of electric field in the paraelectric phase was measured. As an example the field dependence of dielectric constant measured for crystal I at $322.8 \mathrm{~K}$ is presented in Fig. 3. The maximum of $\varepsilon(E)$ dependence is displaced along the $E$-axis. The magnitude of this displacement can be taken as a measure of $E_{\mathrm{B}}$.

If we assume the equivalence of both external and internal bias fields, the state equation (1) should be fulfilled for the net field defined as follows

$$
E_{\text {net }}=E+E_{\mathrm{B}}
$$
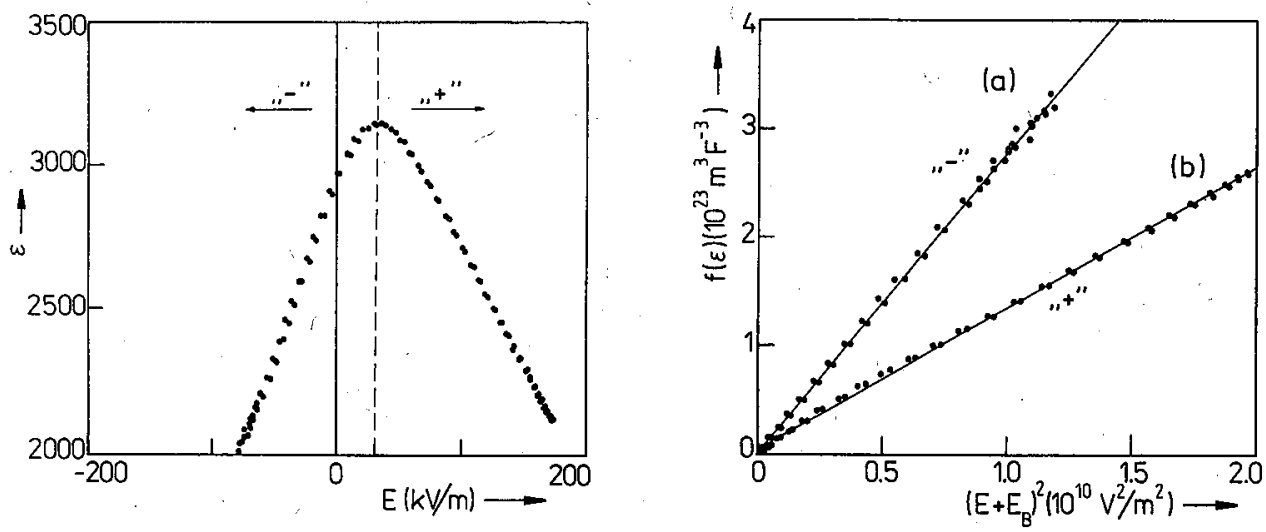

Fig. 3. The dependence of dielectric constant on electric field at $322.8 \mathrm{~K}$ (crystal I). Fig. 4. The field dependence of dielectric permittivity at $322.8 \mathrm{~K}$ (crystal I) according to eq. (4); (a) $E_{\text {net }}$ parallel to $E_{\mathrm{B}}$, (b) $E_{\text {net }}$ antiparallel to $E_{\mathrm{B}}$.

From this point of view the asymmetry of $\varepsilon(E)$ dependence with respect to the axis corresponding $E_{\text {net }}=0$ may appear rather surprising. Such a asymmetry can be seen in Fig. 3 and it was observed in the temperature range of about $2 \mathrm{~K}$ above the phase transition temperature. In the paraelectric phase the state equation (1) generates the following relation for the dielectric permittivity [19]:

$$
\varepsilon^{-3}+3 \varepsilon(0)^{-1} \varepsilon^{-2}-4 \varepsilon(0)^{-3}=27 B E^{2},
$$

where $\varepsilon(0)$ denotes the dielectric permittivity at the zero field. It is understandable that if the internal bias 'exists this relation is satisfied for field $E_{\text {net }}$, and $\varepsilon(0)$ 
(a)

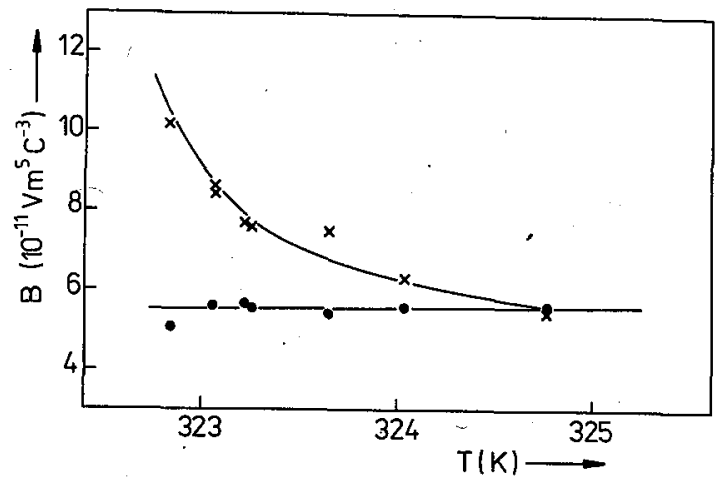

(b)

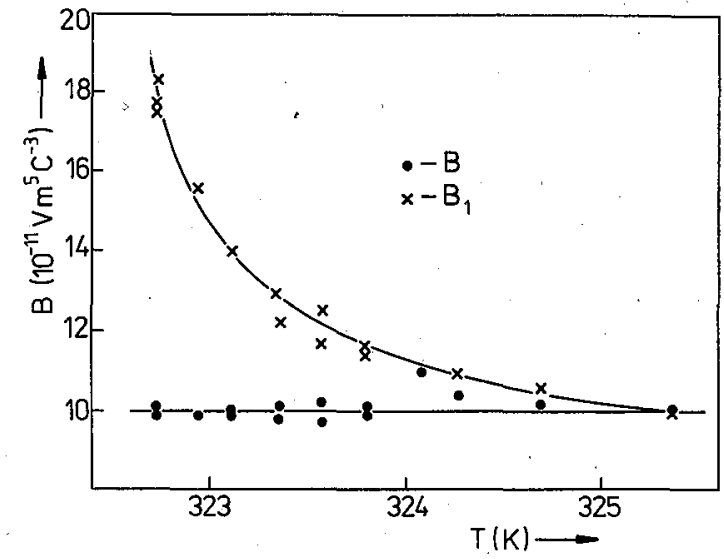

Fig. 5. Temperature dependence of the non-linearity coefficients obtained for crystal I (a) and II (b);. $B$ (for $\left.E_{\text {net }}>0\right), x-B_{1}\left(\right.$ for $\left.E_{\text {net }}<0\right)$.

denotes the maximum value on the $\varepsilon(E)$ curve. The both branches of $\varepsilon\left(E_{\text {net }}\right)$ dependence fulfil equation (4) but with different coefficients $B$ (see Fig. 4).

The non-linearity coefficients obtained for both crystals are presented in Fig. 5 . The $B$ coefficient, determined for $E_{\text {net }}$ of the same sense as $E_{\mathrm{B}}$, increases while approaching the phase transition temperature from the paraelectric phase, whereas for $E_{\text {net }}$ opposite to $E_{\mathrm{B}}$ it is practically temperature independent.

The state equation coefficients can be found in the ferroelectric phase from the temperature dependence of spontaneous polarization. The experimental results obtained for the crystal II are presented in Fig. 6. The solid line is generated from the formula

$$
P_{\mathrm{s}}=\left\{-\frac{B}{2 C}\left[1-\sqrt{1-\frac{4 A_{0} C\left(T-T_{\mathrm{C}}\right)}{B^{2}}}\right]\right\}^{1 / 2},
$$

with $A_{0}=4.1 \times 10^{7} \mathrm{VmC}^{-1} \mathrm{~K}^{-1}, B=10.8 \times 10^{11} \mathrm{Vm}^{5} \mathrm{C}^{-3}, C=9.3 \times 10^{14} \mathrm{Vm}^{9} \mathrm{C}^{-5}$, $T_{\mathrm{C}}=322.54 \mathrm{~K}$. The best-fit parameters $B ; C$ and $T_{\mathrm{C}}$ were found by the least 
squares method, taking for calculations the $A_{0}$ value obtained from the Curie-Weiss law.

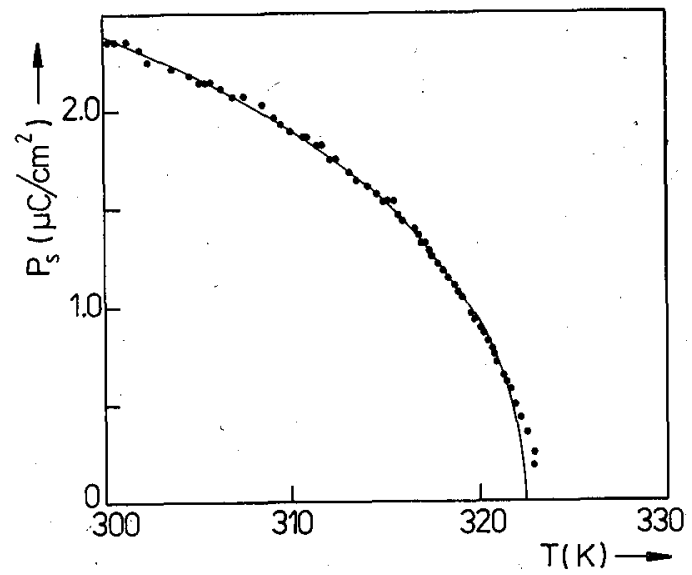

Fig. 6. Temperature dependence of the spontaneous polarization (crystal II). Solid line is calculated for best-fit parameters.

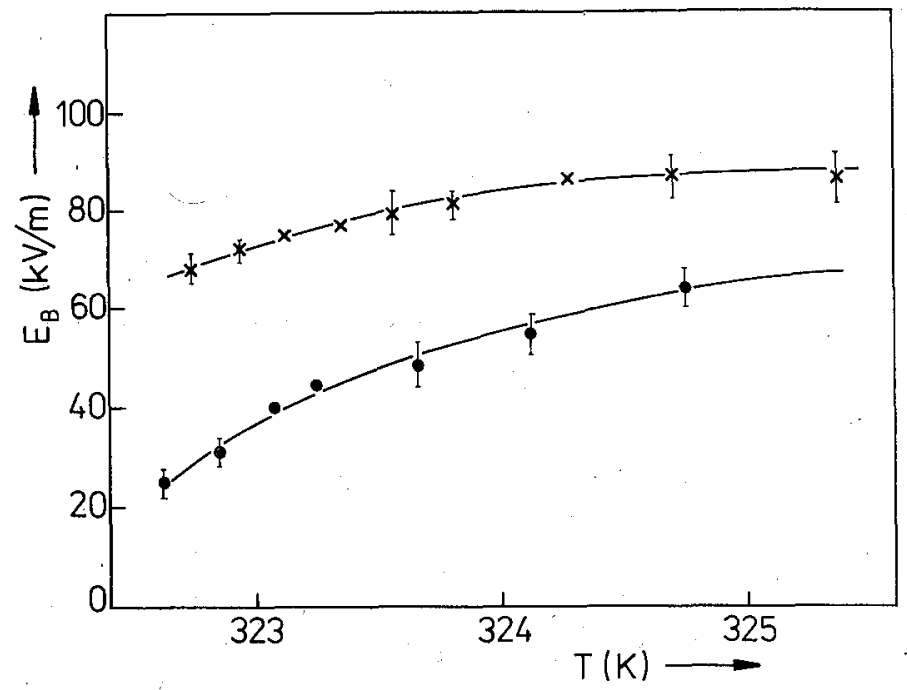

Fig. 7. Temperature dependence of $E_{\mathrm{B}} ; \bullet-$ crystal I, $\mathbf{x}-$ crystal II.

From the position of maximum on the $\varepsilon(E)$ curve, the temperature dependence of $E_{\mathrm{B}}$ in the paraelectric phase was determined. In the case of both crystals the internal bias field decreases while approaching $T_{\mathrm{C}}$ (see Fig. 7).

The changes of $E_{\mathrm{B}}$ were greater in crystal of lower concentration of L-alanine. 


\section{Discussion}

The L-alanine admixture causes local distortion the TGS lattice and substituting for the glycines I it becomes the polar center which will have their dipoles pointing in the same direction. Thus there is a macroscopic irreversible polarization associated with the alanine which polarizes the host. The total dipole moment and the internal bias field due to the alanine in TGS depend not only on the alanine dipole moment but also on the character of interaction between the polar center and the surrounding lattice [20-22]. It seems that for the LATGS of low concentration of L-alanine, the internal bias field depends on temperature [14-16] and to some extent on time [14]. For TGSe crystals with alanine admixture the weak dependence of $E_{\mathrm{B}}$ on temperature in the ferroelectric phase was also found [23]. In all mentioned papers $E_{\mathrm{B}}$ value decreases while approaching the phase transition temperature. It is in agreement with our results.

The obtained values of the dielectric state equation coefficients prove that LATGS cannot be considered as pure TGS at the field $E_{\mathrm{B}}$. The admixtures changing the local fields-electric and mechanic-cause the changes of the state equation parameters. The coefficients obtained for crystal II differ considerably from the ones describing dielectric properties of TGS.

The dependence of the dielectric permittivity on electric field strength in the paraelectric phase demands some additional comments. The dependence asymmetry has been observed earlier for $\gamma$-damaged TGS crystals [24]. L-alanine in TGS crystals generates not only the internal bias field but also the asymmetry of polarizability. The coefficient $B$ obtained for fields $E_{\text {net }}$ opposite to $E_{\mathrm{B}} \cdot("+$ " direction) is temperature independent and approximately of the same value as that resulting from the spontaneous polarization measurements. For $E_{\text {net }}$ parallel to $E_{\mathrm{B}}$ ("-" direction) the nonlinearity coefficient (denoted $B^{\prime}$ ) changes with temperature. Such a temperature dependence seems to be characteristic of crystals whose dielectric properties are influenced considerably by a space charge $[25,26]$. It can be assumed that we deal with some non-ferroelectric effect which gives additional contribution to the non-linear properties of the crystal. The measured dielectric permittivity is the macroscopic quantity which can be influenced by all inhomogeneities of the field in a sample, what can give effect on the $B$ value estimated from $\varepsilon(E)$ dependence. The non-linearity coefficient $B$ appearing in the dielectric state equation cannot depend on the electric field sign, it means that the asymmetry of $\varepsilon(E)$ dependence is of non-ferroelectric origin. However as $B$ obtained for the " + " direction does not depend on temperature and has the value close to that for ferroelectric phase, we can consider it as the "real" value of $B$.

The experiment shows that dielectric behaviour of LATGS crystal can be well described by two state equations

$$
\left.\begin{array}{lll}
E_{\text {net }}=E+E_{\mathrm{B}}=A_{0}\left(T-T_{\mathrm{C}}\right) D+B D^{3} & \text { for } & E_{\text {net }}>0 \\
E_{\text {net }}=E+E_{\mathrm{B}}+A_{0}\left(T-T_{\mathrm{C}}\right) D+B_{1} D^{3} & \text { for } & E_{\text {net }}<0
\end{array}\right\} .
$$

Assuming that $B$ is the non-linearity coefficient for the both equations, we are in agreement with the experiment providing that $E_{\mathrm{B}}$ depends on the electric field. 
Thus the equations can be formulated as follows:

$$
\left.\begin{array}{lll}
E+E_{\mathrm{B}}=A_{0}\left(T-T_{\mathrm{C}}\right) D+B D^{3} & \text { for } & E_{\text {net }}>0 \\
E+\left(E_{\mathrm{B}}+\Delta E_{\mathrm{B}}\right)=A_{0}\left(T-T_{\mathrm{C}}\right) D+B_{1} D^{3} & \text { for } & E_{\text {net }}<0
\end{array}\right\}
$$

Hence $\Delta E_{\mathrm{B}}=\left(B_{1}-B\right) D^{3}$.

Taking $D(E)$ from the first equation we can calculated the changes of the internal bias field $\Delta E_{\mathrm{B}}$ caused by external electric field. The dependence of $E_{\text {net }}$ on external electric field calculated for $T=T_{\mathrm{C}}+0.2 \mathrm{~K}$ is presented in Fig. 8. For $E_{\text {net }}<0$ there is a deviation from the linear relationship between $E_{\text {net }}$ and

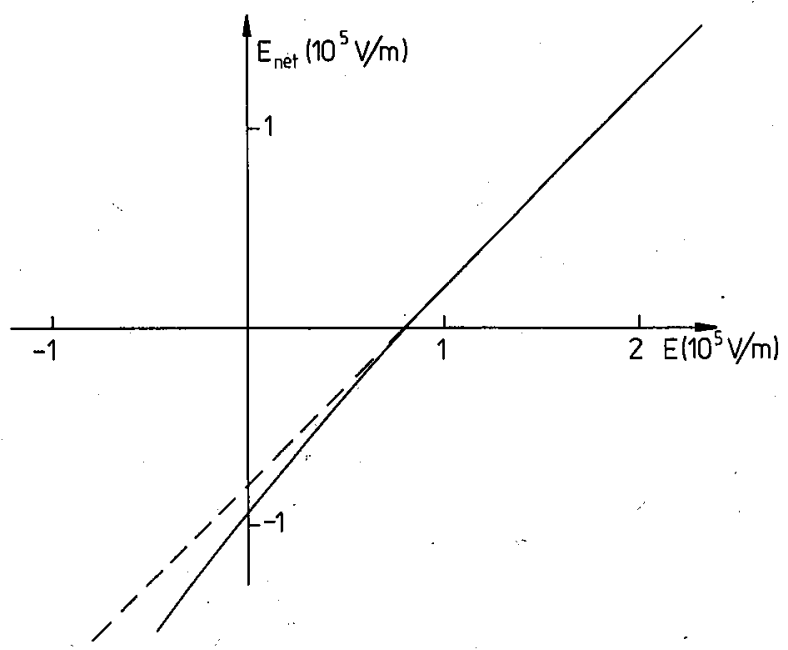

Fig. 8. The net field $E_{\text {net }}$ as a function of external electric field at $T_{\mathrm{C}}+0.2 \mathrm{~K}$ calculated for crystal II.

$E$. The internal bias field dependence on $E$ is presented in Fig. 9 for two chosen temperatures. (It should be mentioned that such à dependence was obtained after several repolarization of the sample.)

Thus the asymmetry of the non-linearity of LATGS can be attributed to the field dependence of internal bias.

Considering the presented results it is hard to explain a mechanism of the internal bias field behaviour. However, it seems likely that internal field produced by L-alanine is to some extent screened by the space charge.

Description of LATGS dielectric properties by the classical state equation can be regarded only as the first approximation. Theoretical treatment of a ferroelectric crystal with polar anisotropy centers has been proposed by Okada [27]. The theory . has been used for LATGS properties discussion by Tylczyński [28]. In this approach the internal bias field is expressed by the, formula

$$
E_{\mathrm{B}}=-(1-\lambda) k P_{0} /(1+a k)
$$




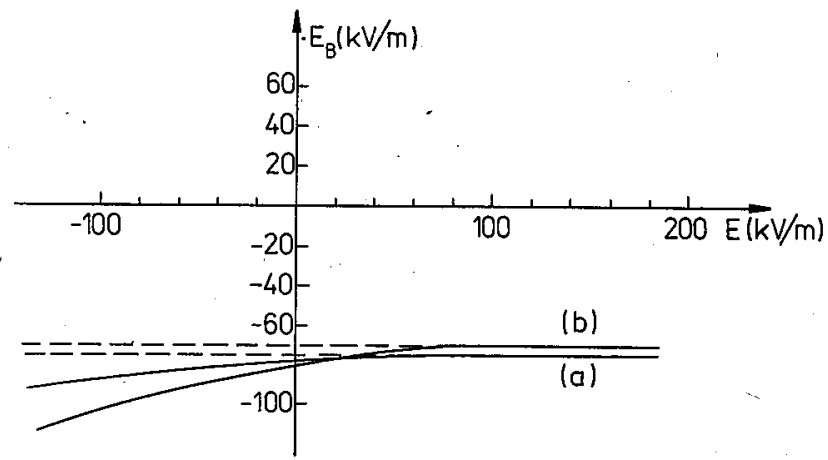

Fig. 9. The internal bias field dependence on external electric field calculated for crystal II; $T-T_{\mathrm{C}}=0.5 \mathrm{~K}(\mathrm{a})$ and $0.2 \mathrm{~K}(\mathrm{~b})$.

where: $\lambda$ is the volume fraction of the ferroelectric part in the crystal, $P_{0}-$ polarization of the region occupied by polar anisotropy centers without the electric field, $k$ - the interaction constant between the ferroelectric region and that occupied by polar centers, $a$ - dielectric susceptibility of that second region. $P_{0}$ is a factor which depends not only on the alanine dipole moment but also on the displacement of the charge in the surrounding lattice. It seems that the charge distribution around the glycine admixture is modified by external electric field but this effect depends on the electric field direction.

\section{References}

[1] P.J. Lock, Appl. Phys. Lett. 19, 390 (1971).

[2] E.T. Keve, K.L. Bye, P.W. Whips, A.D. Annis, Ferroelectrics 3, 39 (1971).

[3] K.L. Bye, W. Whipps, E.T. Keve, Ferroelectrics 4, 253 (1972).

[4] M. Koralewski, J. Stankowska, T. Jasiński, Jpn. J. Appl. Phys. 26, 383 (1987).

[5] J. Stankowska, T. Jasiński, Acta Phys. Pol. A71, 959 (1987).

[6] K. Imai, H. Ishida, Ferroelectrics 106, 321 (1990).

[7] J. Del Cerro, S. Ramos, Jpn. J. Appl. Phys. 24, 341 (1985).

[8] F. Jiménez, S. Ramos, J. Del Cerro, Ferroelectrics 92, 369 (1989).

[9] H. Pykacz, J. Mróz, Ferroelectr. Lett. 2, 73 (1984).

[10] B. Brezina, M. Havrankova, Cryst. Res. Technol. 20, 787 (1985).

[11] N. Nakatani, Jpn. J. Appl. Phys. 29. 2774 (1990).

[12] M.A. Gaffar, G.F. Al-Noaimi, A. Abu El-Fadl, J. Phys. Soc. Jpn: 58, 3392 (1989).

[13] J.L. Martinez, A. Cintas, E. Diequez, J.A. Gonzalo, Ferroelectrics 44, 221 (1983). 
[14] F. Jiménez, S. Ramos, J. Del Cerro, Phase Transit. 12, 275 (1987).

[15] F. Jiménez, S. Ramos, J. Del Cerro, Ferroelectrics 94j 443 (1989).

[16] S. Ramos, F. Jiménez, J. Del Cerro, Ferroelectrics 108, 319 (1990).

[17] H. Schmitt, H.E. Müser, O. Mengelkoch, W. Sterta, Ferroelectrics 25, 499 (1980).

[18] J. Bornarel, V.H. Schmidt, J. Phys. C: Solid State Phys. 14, 2017 (1981).

[19] R. Cach, A. Jaśkiewicz, R. Lamber, Acta Phys. Pol. A62, 249 (1982).

[20] V.K. Novik, N.D. Gavrilova, G.T. Galstyan, Kristallografiya 28, 1165 (1983).

[21] M.F. Lines, A.M. Glass, Principles and Applications of Ferroelectrics and Related Materials, Claredon Press, Oxford 1977.

[22] B. Hilczer, Cz. Pawlaczyk,KTB Martin-Luther-Universität (0 21), 71 (1986).

[23] J. Stankowska, Z. Trybuła, Acta Phys. Pol. A62, 457 (1982).

[24] R. Cach, Ferroelectr. Lett. 6, 85 (1986).

[25] R. Cach, S. Dacko, Acta Phys. Pol. A76, 521 (1989).

[26] R. Cach, J. Jakubas, Ferroelectrics 108, 121 (1990).

[27] K. Okada, J. Phys. Soc. Jpn. 16, 414 (1961).

[28] Z. Tylczyński, Acta Phys. Pol. A58, 275 (1980). 